\title{
MS06-01 | Structural Studies of the Leading Strand DNA Polymerase in
}

\section{EUKARYOTES}

Johansson, Erik (Umeå University, Umeå, SWE)

In eukaryotes, the duplication of the genome requires three different DNA polymerases with separate functions. Both genetic experiments and biochemical experiments have shown that DNA Polymerase epsilon is responsible for the bulk synthesis of the leading strand during DNA replication. In addition, it was recently shown that a mutation in the exonuclease domain of Pol epsilon, leads to an impaired proofreading capacity and is responsible for a very high mutation load in sporadic tumors. We have in addition to biochemical and genetic experiments solved a series of ternary structures of the catalytic core of Pol2 (142 kDa), the catalytic subunit of yeast Pol epsilon. I will here discuss some of our most recent findings, for example the structural basis for the very high processivity of DNA polymerase epsilon, the presence of an Fe-S cluster in the catalytic domain, and how a single amino acid substitution (P301R in yeast, orthologous to P286R in human Pol epsilon) can alter the high-fidelity polymerase to become a very strong mutator polymerase. The structure, in combination with molecular dynamics simulations, revealed major differences between Pol epsilon - P301R and the catalytically inactive Pol epsilon D290A,E292A in the mechanism of exonuclease inactivation, and help explain the vastly stronger mutagenic and tumorigenic effects of the cancer variant. 